\title{
Participación popular y política de desarrollo urbano en Cruz de Motupe
}

Recibido: 16/03/2020

Aprobado: 04/05/2020

Hoffmann Ticllacuri Meza

Publicado: 25/08/2020

Universidad Nacional Mayor de San Marcos

$<$ htm_1110@hotmail.com>

\begin{abstract}
RESUMEN
La complejidad de los proyectos de infraestructura social ha sido la expresión de un desarrollo inconcluso, como es el caso de la construcción de pistas y veredas en Cruz de Motupe ${ }^{1}$ durante el 2011. El objetivo de la investigación es explorar el desenvolvimiento de la participación del sector popular en la gestión de políticas de obras públicas, desde la sombra de un patrón de poder municipal que se ha articulado dentro del aparato del Estado. La metodología se ha realizado mediante el acceso a los estatutos, fotos y documentos de inversión pública, provenientes de la Municipalidad de San Juan de Lurigancho y del Programa Integral de Mejoramiento de Barrios y Pueblos (PIMBP) del Ministerio de Vivienda, Construcción y Saneamiento (MVCS).
\end{abstract}

Palabras clave: Desarrollo, participación, sector popular, patrón de poder municipal y Estado.

\section{Popular participation and urban development policy in Cruz de Motupe}

\begin{abstract}
The complexity of the social infrastructure projects has been the expression of an unfinished development, as is the case of the construction of tracks and sidewalks in the young town of Cruz de Motupe during 2011. The objective of the research seeks to explore the development of the participation of the popular sector in the management of public works policies under the shadow of a pattern of municipal power that has been articulated within the state apparatus. The methodology has been carried out by accessing the statutes, photos and public investment documents from the Municipality of San Juan de Lurigancho and the Integral Program for the Improvement of Neighborhoods and Towns (PIMBP) of the Ministry of Housing, Construction and Sanitation.
\end{abstract}

KeYwords: Development, Participation, Popular sector, Pattern of municipal power and the State.

1 Se encuentra ubicado en la comuna N.o 16, en el distrito de San Juan de Lurigancho, Lima, Perú. 


\section{Introducción}

C ruz de Motupe $^{2}$ es un pueblo joven que ha experimentado cambios a nivel estructural, a partir de los procesos históricos y sociales que se han desarrollado durante los últimos tiempos.

Desde la década de los ochenta, las organizaciones populares en las barriadas han aumentado, de manera considerable, por su heterogeneidad. Los dirigentes populares se radicalizaron por la incidencia de organizaciones políticas de tendencia izquierdista para canalizar demandas y exigencias mediante luchas populares. En ese sentido, Cruz de Motupe no es ajena a esta situación, en 1983 formó la primera comisión proterreno, gracias a la conformación de Comités de Manzana, para exigir las necesidades de lotes de terreno mediante acciones colectivas, frente a un modelo dominante que acarreaba el aislamiento social y la pobreza.

Posteriormente, en los años noventa, el diseño de las políticas de obras públicas de los programas sociales del Estado fue formulado sin ninguna consulta popular, y la selección de proyectos fue dirigida por autoridades políticas, asesores o donantes que estuvieron alejados de las demandas y las necesidades de las masas populares, las cuales fueron relegadas en la toma de decisiones. Estos hechos fueron aprovechados por los agentes externos que manipulaban los tipos de proyectos de obras públicas para intereses particulares, lo que los hizo caer en una profunda acción clientelar y ser parte de un sistema de manipulación para mantener la pasividad de las organizaciones populares. En el caso de Cruz de Motupe, la participación de los sectores populares, en la gestión de obras públicas, perdió capacidad organizativa y sentido crítico, como resultado de la entrega asistencialista de servicios públicos, por ejemplo, de luz, agua y desagüe. Es decir, la participación popular ingresó en una esfera agonizante para abrirles paso a las lógicas individualistas.

Luego, en el 2000, se intentaron reorganizar y rediseñar las políticas de desarrollo para generar nuevos vínculos de participación con los sectores populares, en la toma de decisiones, con un enfoque de derechos. Al respecto, fue indispensable fortalecer la Carta Social para promover los espacios y los canales

2 Se fundó el 14 de octubre de 1984. de participación. Sin embargo, la participación popular, en Cruz de Motupe, se ha desplazado hacia un proyecto de modernidad que impone una participación privatizada, la cual intenta dejar a un lado el sentido de la vecindad.

En esa medida, entre el 2001 y el 2006 se impulsó la creación de instrumentos para mejorar la administración de los recursos de las políticas de desarrollo, uno de ellos fue el Sistema Nacional de Inversión Pública (SNIP). Cabe destacar que el Estado empezó a transferir responsabilidades a los gobiernos locales, con la intención de que los recursos y el gasto público fueran mejor utilizados, no obstante, la visión tecnocrática había llenado los vacíos políticos y sociales. De cualquier forma, la participación popular en la gestión de políticas de desarrollo fue menor, pues fue seducida por la facilidad clientelista que puso obstáculos a la desafiante cultura participativa popular. Además, el comportamiento patrimonial de las autoridades políticas y de los funcionarios del Estado no había ofrecido eficientes garantías y mecanismos de participación en un medio de alta inconsistencia popular.

Más adelante, entre el 2006 y el 2011, la vinculación del Estado y las organizaciones populares se tornó en un escenario conflictivo y represivo, al dejar de promover la participación popular que se había atomizado por su alta complejidad social. Es así como la participación ha supervivido de manera anacrónica y ha entrado en un desborde debido a los actores políticos que se adentran en un proceso de competencia para obtener respaldo o legitimidad de las organizaciones populares. En ese mismo contexto se crearon políticas de desarrollo para aliviar la pobreza, por ejemplo, el PIMBP, mediante el Decreto Supremo N. ${ }^{\circ}$ 017-2007-Vivienda para proveer la ejecución de las obras de infraestructura en sectores populares que sean indispensables en la vida del vecindario, tales como las pistas o las calzadas, las vías, los puentes, los parques y obras similares, en coordinación con los gobiernos locales. De este modo, se cree que estos esfuerzos han llevado a una participación popular incipiente, aplastada por la esfera consumista que se globalizado en una relación de dominación.

Por último, entre 2011 y 2016, se intentó lograr el crecimiento a partir de un enfoque de inclusión social. Así pues, la implementación de múltiples políticas de desarrollo ha traído consigo la masificación de 
las organizaciones populares en los nuevos espacios urbanos, lo que ha ocasionado un modelo dependiente que impulsa el Estado. Se hace necesario resaltar que Cruz de Motupe cambió su denominación de asentamiento humano a pueblo joven en septiembre del 2014, al asumir la integridad de sus obligaciones y la totalidad de los derechos que le corresponden. Sin embargo, su débil capacidad organizativa ha condicionado la superioridad de la gestión municipal para ordenar y ejecutar proyectos de infraestructura social.

\section{Referentes teóricos conceptuales}

\subsection{Desarrollo local}

El desarrollo local en espacios populares ha sido insatisfecho o permanece inconcluso, debido al uso coercitivo de la burocracia estatal, el cual garantiza intereses particulares y atenta contra las reglas de carácter formal. En este sentido, el control deficiente de los dirigentes, de los funcionarios y de las autoridades locales no ha contribuido a viabilizar políticas proclives y eficientes para impulsar la participación popular. Su incidencia en la vida social ha resultado una estafa o destrucción, pues es una forma de discurso político del patrón colonial moderno (Quijano, 2014: 848). Más aún, su proceso deliberado atomiza las relaciones populares desde un esquema interdependiente para amenazar las raíces históricas (Ríos, 2011: 109).

\subsection{Estado}

El Estado transfiere recursos a los gobiernos locales, sin embargo, el mal uso de estos ha desencadenado sobornos y malversación de fondos. La crisis que enfrenta el Gobierno se debe a que las organizaciones políticas no han cumplido un rol central en la confección de una agenda pública que tenga en cuenta la participación popular en la gestión de las políticas de desarrollo y en la toma de decisiones de los asuntos gubernamentales. En este orden de ideas, hay un desprestigio de la política y de sus actores (Lynch, 2017: 30). Por ello, se cree que el Estado, como aparato tecnócrata, se ha alejado de la vida popular, puesto que su comportamiento es excluyente; la única forma de encontrar un verdadero espacio deliberativo es el reconocimiento de la participación popular, en un horizonte de debate de racionalidad ética, ${ }^{3}$ para orientar los criterios gubernamentales.

\subsection{Política de desarrollo}

La gestión de políticas de desarrollo se relaciona con las interacciones entre el Estado y las organizaciones populares para la determinación de disposiciones orientadas al bienestar (Alza, 2008: 54). Este conjunto de criterios encamina la conducta del Estado al mejoramiento de los modos de vida, y supone esquemas valorativos, funcionales, políticos institucionales, históricos, normativos y legales (Béjar, 2007: 34). Además, su desenvolvimiento es producto de la acción contradictoria de distintas fuerzas populares que pueden ser beneficiosas o perjudiciales para la forma de vida de las personas.

\subsection{Municipio}

El Estado brinda recursos a los gobiernos locales para el buen uso social, político, económico y cultural. No obstante, ha sido capturado por el mal uso del poder político burocrático, mediado por grandes intereses, a través de la malversación de recursos, mientras se expande a los diferentes espacios de la vida pública (Quiroz, 2013: 30). En tal sentido, el municipio se ha convertido en una agencia gubernamental del Estado para la prestación de servicios públicos a los sectores populares, pero su percepción ha sido clandestina ${ }^{4}$, lo que conlleva la desintegración social y política (Mejía, 1990: 79). En la actualidad, esta intenta revalorarse como una entidad notoria y visible de estabilidad y modernidad, a pesar de privilegiar grupos poderosos en el manejo ilegal de obras públicas y de caer en acciones clientelares; por el momento, no ha existido una modificación racional en su estructura orgánica ni en los procedimientos con los que opera (Mejía, 1990: 88).

Para tal efecto, se cree que el municipio pierde su protagonismo en la vida social, al distanciarse de las

3 La racionalidad ética se refiere a la interacción y reconocimiento histórico de los sujetos sociales en la responsabilidad de las acciones o cuestiones prácticas para una cultura de vida auténticamente humana. Puede consultarse a Josefina Fantoni: Racionalidad ética. Una acción responsable, Jujuy, Argentina, 2002.

4 La clandestinidad se refiere a zonas ocultas de ilegalidad que encarnan la tensión entre inclusión-exclusión, visibilidad-invisibilidad y lo conocido-negado. Puede consultarse a Bárbara Sutton: Zonas de clandestinidady "nuda vida": mujeres, cuerpo y aborto, Santa Catarina, Brasil, 2017. 


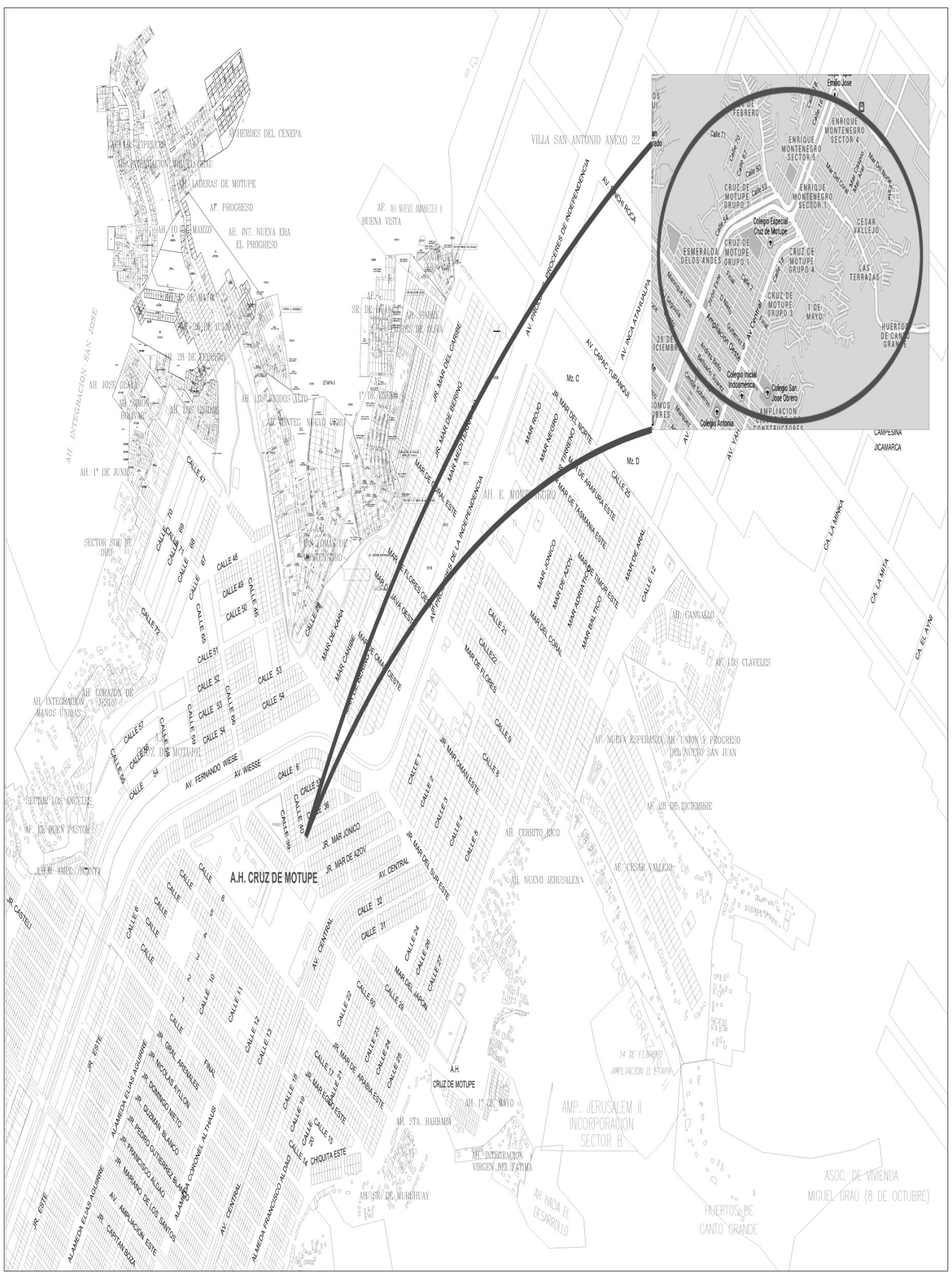

Figura 1: Ruta de acceso al pueblo joven Cruz de Motupe (2020) 
demandas y las exigencias populares, y al satanizarlas con obras asistencialistas conducidas por funcionarios quienes manejan a su favor intereses particulares, y dejan a un lado las acciones colectivas. Aunque este ha existido desde antes que el Estado, la mala asignación de fondos ha debilitado su existencia institucional, lo que provoca una burbuja de patronazgo (Quiroz, 2013: 39). La burocracia ha separado los sectores populares del municipio, al ser una institución sensible que adolece de serias dificultades en su estructura administrativa (Mejía, 1990: 115).

\subsection{Participación popular}

La participación popular ha sido efímera y dependiente cuando se ha enfocado en alcanzar logros específicos, al interior de un ciclo temporal que ha emergido como práctica significativa, pero es apreciada de forma genérica, sin un verdadero análisis de sus problemas o limitaciones en las políticas de desarrollo (Tanaka, 2001: 17). Al respecto, las organizaciones populares no han llegado a consolidarse social ni políticamente por estar sometidas a intereses dominantes que han lesionado la vida colectiva. De esta forma, la demanda popular se inscribe en una lógica de conflictividad de luchas entre distintos actores (Panfichi y Coronel, 2014: 13).

\subsection{Los sectores populares}

Los sectores populares se desenvuelven en una esfera consumista, como producto de la transformación de la sociedad (Mejía, 2014: 53). Su conjunto heterogéneo se encuentra regido por relaciones de subordinación (Quijano, 2014: 106). De ahí se desprende que la exaltación de formas individualistas, en un contexto dependiente, ha traído consigo el deterioro social de la organización popular y la existencia de nuevas lógicas, códigos y símbolos.

\section{Pueblo joven Cruz de Motupe}

\subsection{Ubicación}

Se encuentra ubicado en la comuna N. ${ }^{\circ} 16$, distrito de San Juan de Lurigancho, Lima. Está encerrado dentro de los siguientes linderos: por el norte colinda con los pueblos jóvenes Enrique Montenegro, Los Unidos y Virgen de Fátima; por el sur con el asentamiento humano Mariscal Cáceres; por el este con el pueblo joven $1 .^{\circ}$ de mayo y el asentamiento humano Los Unidos; y por el oeste con los pueblos jóvenes Corazón de Jesús y 10 de marzo, y el asentamiento humano Simón Bolívar (Fuente: Estatuto, 2014).

La ruta de ingreso se realiza por la Av. Próceres de la Independencia que conecta con la Av. Fernando Wiesse, por las cuales transitan líneas de combis y micros. También se puede llegar por dos vías paralelas de menor capacidad vehicular, como las avenidas Central y Canto Grande.

\subsection{Contexto del barrio}

Tiene como sede institucional el Local Comunal, que cuenta con un área de $3000 \mathrm{~m}^{2}$ y se ubica frente a la Plaza Mayor de la misma comunidad. Esta población se divide en siete grupos habitacionales con diferentes problemáticas, a causa de su alta complejidad social.

\subsection{La organización popular}

\subsection{Los niveles de organización}

El primer nivel de organización lo constituyen las calles, integrada por los pobladores de los frentes de manzana, cuya fachada se sitúa hacia una misma calle por cuadra. El segundo nivel de organización lo constituye el grupo habitacional, conformado por miembros que residen en un lugar en común. El tercer nivel está formado por la integración de los comités de calles, Comité de Gestiones, Comité de Seguridad Ciudadana, Comité de Parque y las juntas directivas de los siete grupos habitacionales, además de todas las organizaciones existentes en la organización vecinal; tiene como representante legal permanente a la Junta Directiva Central (JDC). El cuarto nivel superior es la Asamblea General, al ser la máxima instancia.

\subsection{Los órganos del sistema}

\subsection{1. Órganos de decisión}

La Asamblea de Calles (AC) es el primer nivel de decisión resolutiva, el cual se reúne al ser convocado por 
TABla 1. Características de los grupos habitacionales

\begin{tabular}{|c|c|}
\hline Grupo 1 & $\begin{array}{l}\text { A diferencia de los otros grupos habitacionales, este tiene una reducida extensión territorial. Existe desorganiza- } \\
\text { ción en cuanto a su Junta Directiva de Grupo para enfrentar problemas de pandillaje y drogadicción en su losa } \\
\text { deportiva. Otra problemática registrada es el uso del Local Comunal para fines mercantiles. Cuenta con dos } \\
\text { instituciones relevantes: el centro de atención infantil Cuna Más y el Club de Madres María Parado de Bellido. }\end{array}$ \\
\hline Grupo 2 & $\begin{array}{l}\text { Su local comunal opera como un pequeño parque de juegos en precarias condiciones de infraestructura. En } \\
\text { sus calles internas se observan instituciones, como el nido de guardería Mundo Kids, la institución educativa } \\
\text { N. }{ }^{\circ} 105 \text { Yoy Marina Gárate Bardales y el comedor Cruz de Motupe. }\end{array}$ \\
\hline Grupo 3 & $\begin{array}{l}\text { Tiene espacios abandonados y su local comunal funciona como vivienda de personas que habían comprado a } \\
\text { los dirigentes en años anteriores. Otra problemática es el alquiler de cochera (car wash) para fines personalistas. } \\
\text { En ese espacio se iba a construir un Programa no escolarizado de Educación Inicial (Pronoi), que no se llegó } \\
\text { a ejecutar, y ahora funciona en el colegio Micaela Bastidas N. } \text { o }^{151} \text {, que fue fundado el } 8 \text { de marzo de } 1985 \text {. } \\
\text { En sus calles internas se observan algunas instituciones, por ejemplo, el comedor popular Corazón de Jesús, la } \\
\text { capilla Santísima Cruz de Motupe N.o } 3 \text { y el colegio privado Sol Naciente. }\end{array}$ \\
\hline Grupo 4 & $\begin{array}{l}\text { Se fundó en } 1986 \text { y en la actualidad consta de } 350 \text { lotes de vivienda, en un promedio de } 120 \mathrm{~m}^{2} \text {. Su Complejo } \\
\text { Deportivo No } 1 \text { tiene una extensión de } 24153.05 \mathrm{~m}^{2} \text { y se encuentra en condiciones deplorables con montículos } \\
\text { de basura, además, no cuenta con iluminación y eso estimula la presencia de extrańos para ingerir bebidas alco- } \\
\text { hólicas, fumar y, en algunos casos, drogarse; a pesar de que entró en un proceso de mejoramiento en el } 2017 \text { por } \\
\text { la Unidad Formuladora del Gobierno Local, con un monto de } 3672193.43 \text { nuevos soles y al beneficiar a } 4340 \\
\text { moradores. En ese mismo año se impulsó la construcción del parque N. } 4 \text { y el remodelado de la losa deportiva, } \\
\text { cuyo monto ascendió a la suma de } 652973.78 \text { nuevos soles, en la gestión del alcalde Juan Navarro Jiménez. } \\
\text { Por otra parte, en sus calles internas se encuentran los colegios privados Orión School, Nivel A, Tradicionista } \\
\text { Ricardo Palma y Santa Inés. Igualmente, está la institución estatal N.o } 115-13 \text { Ugel } 05 \text { Virgen de las Mercedes, } \\
\text { así como el Club de Madres María Auxiliadora, que ha desempeñado un papel importante en la distribución de } \\
\text { raciones a las personas carentes de recursos. }\end{array}$ \\
\hline Grupo 5 & $\begin{array}{l}\text { Se estima en } 3125 \text { habitantes distribuidos en } 639 \text { viviendas, con un promedio de cinco personas por vivienda. } \\
\text { Fue un grupo muy organizado, a diferencia de los otros, pero ahora intenta consolidar su posicionamiento. Su } \\
\text { local comunal se encuentra abandonado y con poca iluminación. Las personas cercanas aprovechan ese espacio } \\
\text { para defecar y raras veces atrae la presencia de sujetos para drogarse; entretanto, su losa deportiva constituye } \\
\text { un espacio social en donde se realizan las asambleas de grupo, que en la actualidad es conservada por un grupo } \\
\text { de jóvenes que se hace llamar Sport Unión, el cual ha reflexionado sobre la importancia de mantener en orden } \\
\text { sus cuadras y calles. } \\
\text { En sus calles internas se observa el impulso del comercio ambulatorio popular, específicamente en la avenida } \\
\text { conocida como La Curva, que a la vez alberga la concentración de motos de la Asociación de Motupe Nueva } \\
\text { Imagen. También, se aprecian instituciones como la iglesia cristiana misionera Vida Abundante, el colegio pri- } \\
\text { vado Montefiory, el club de madres Señor de los Milagros, la capilla Cruz de Motupe, el centro de salud Cruz } \\
\text { de Motupe y el centro infantil de atención integral Cuna Más. }\end{array}$ \\
\hline Grupo 6 & $\begin{array}{l}\text { Su complejo deportivo N. } 2 \text { fue fundado el } 8 \text { de mayo del 2003, con partida N.o } 13442831 \text {. En sus ambientes } \\
\text { internos se percibe cancha de grass, juego para nińos y losas deportivas. Cerca de la asociación deportiva existe } \\
\text { un vivero de plantas. Entre las instituciones educativas privadas destacan: la Institución Educativa Gran Amau- } \\
\text { ta, } 9 \text { de Diciembre y Bienvenidos a su casa del saber. Mientras que a nivel estatal se encuentra el colegio inicial } \\
\text { N. }{ }^{\circ} 115-6 \text { Los Angelitos. Su local comunal opera como vivienda posesionaria; esta situación ocurre también } \\
\text { cerca al comedor Nińos de La Paz. }\end{array}$ \\
\hline Grupo 7 & $\begin{array}{l}\text { Cuenta con una extensión aproximada de } 165441.51 \mathrm{~m}^{2} \text {, de las cuales } 52941.28 \mathrm{~m}^{2} \text { están destinadas a áreas de } \\
\text { circulación, cuya cobertura de pavimentación es del } 15 \% \text {. En cuanto al sector comercial, en el Mercado } 11 \text { de } \\
\text { enero se percibe un fuerte comercio ambulatorio, en donde los sectores populares entretejen nuevas relaciones } \\
\text { sociales ante un modelo económico dependiente. En sus calles internas están el club de madres María Mazza- } \\
\text { rello, el Club de Madres Niño Jesús, el centro infantil de atención integral Cuna Más, el colegio privado Niño } \\
\text { Manuelito, el colegio Túpac Amaru II y el jardín inicial Niño Jesús. Además, cuenta con un espacio para un } \\
\text { taller artesanal, que en la actualidad opera de manera ilegal como lugar de viviendas. Su local comunal se con- } \\
\text { centra cerca de Cuna Más de Cruz de Motupe, pero las reuniones de asambleas se realizan de manera esporádica } \\
\text { en la losa deportiva de la parroquia San José Obrero. }\end{array}$ \\
\hline
\end{tabular}

Fuente: elaboración propia 
el delegado de calle; ordinariamente cada seis meses y extraordinariamente todas las veces que la organización lo requiera. El segundo nivel de decisión es la asamblea del grupo habitacional; sus decisiones solo podrán ser observadas y revocadas por las instancias superiores de la organización, como la JDC y la asamblea general de pobladores. Esta se reúne ordinariamente cada tres meses y extraordinariamente todas las veces que las necesidades de la organización lo requieran; es convocada y dirigida por el coordinador de grupo, con conocimiento y asistencia de la JDC. El tercer nivel de decisión resolutiva es la asamblea de la JDC que se reúne cada treinta días calendario y extraordinariamente con la participación plena de la JDC; las citaciones se realizan con una anticipación no menor de dos días. Por último, el cuarto nivel de decisión es la Asamblea General, que es el órgano supremo y está conformado por todos los pobladores motupeños inscritos en el libro padrón de pobladores.

\subsubsection{Dirección ejecutiva}

La JDC es la máxima instancia en el sistema de Dirección Ejecutiva. Es constituida por elecciones generales mediante voto secreto y universal, por el período de dos años; cabe resaltar que en el cargo de presidente no se puede reelegir a la misma persona de forma consecutiva. Las acciones de la JDC son observadas por el órgano de control de fiscalización en un plazo máximo de ocho días, caso contrario se traslada para ser tratado en la Asamblea General del pueblo.

\subsubsection{El Comité Electoral}

Es autónomo en sus funciones y es elegido por un período de dos años. Entre sus funciones están: elaborar todo proceso electoral, producir cédulas de sufragio y juramentar a la nueva dirigencia.

\subsubsection{Comité de Seguridad Ciudadana}

Representa a los vecinos y tiene como objetivo proteger a la población de la inseguridad. Se elige en una asamblea general por un período de dos ańos. Se encuentra conformado por cinco miembros: el presidente, el vicepresidente, el secretario de actas, el secretario de economía y el vocal. En la práctica su incidencia es débil.

\subsubsection{Comité de Parque}

Es una organización encargada de velar por la conservación adecuada del área verde. Se conforma gracias a una asamblea de grupo habitacional, por un periodo de dos años. Se compone de cinco miembros, con los siguientes cargos: presidente, vicepresidente, secretario de actas, secretario de economía y vocal.

\subsubsection{Consejo de Fiscalización}

Es el órgano de control interno de la organización, cuya finalidad es ejercer la fiscalización de los actos de la JDC y de las comisiones de toda organización social existente. Se encuentra integrado por tres miembros: presidente, secretario y vocal. Sin embargo, en la práctica su rol es inexistente.

\section{La gestión municipal}

La Municipalidad de San Juan de Lurigancho no ha culminado la construcción de pistas y veredas en el pueblo joven Cruz de Motupe, por lo que tiene calles polvorientas y en mal estado, lo que ha ocasionado accidentes, en su mayoría de gravedad.

Si se realiza un examen exhaustivo, se puede decir que la ejecución de la obra pública en la gestión de Carlos Burgos Horna (2007-2014) ha sido una política inconclusa en la población, puesto que se desarrolla en un contexto de burocracia pública al violar las normas legales, por ejemplo, el delito de enriquecimiento ilícito y lavados de activos en agravio con el Estado. Su incidencia ha operado en el marco del poder municipal para subordinar la organización popular de los motupeńos en el proceso de fiscalización de la obra pública, con lo cual amenazó las raíces históricas del pueblo.

Esta visión eurocéntrica ha deslegitimado la negación del otro como parte de un patrón colonial de poder en una cuestión de dependencia histórica (Quijano, 2009: 850). Asimismo, la gestión edil ha afectado la confianza del sector popular, para dar paso a redes de poder no formales. La transgresión del municipio ha logrado posicionarse como un instrumento del sistema central, para otorgar algunas obras públicas con orientación asistencialista a los sectores populares, y al marcar distancia de la cultura participativa en el proceso de control y fiscalización. De esta forma, sus maniobras han sido clandestinas 


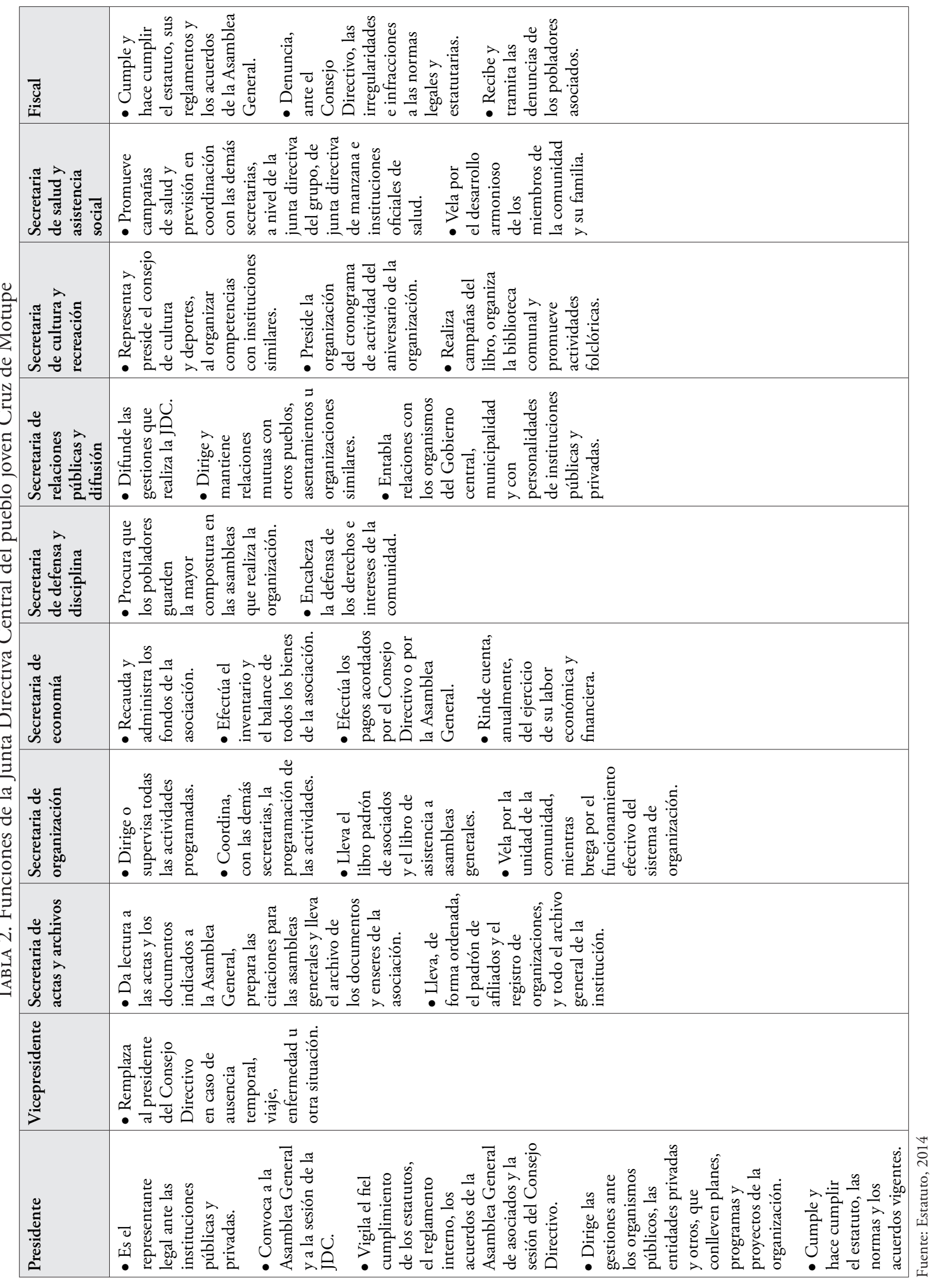




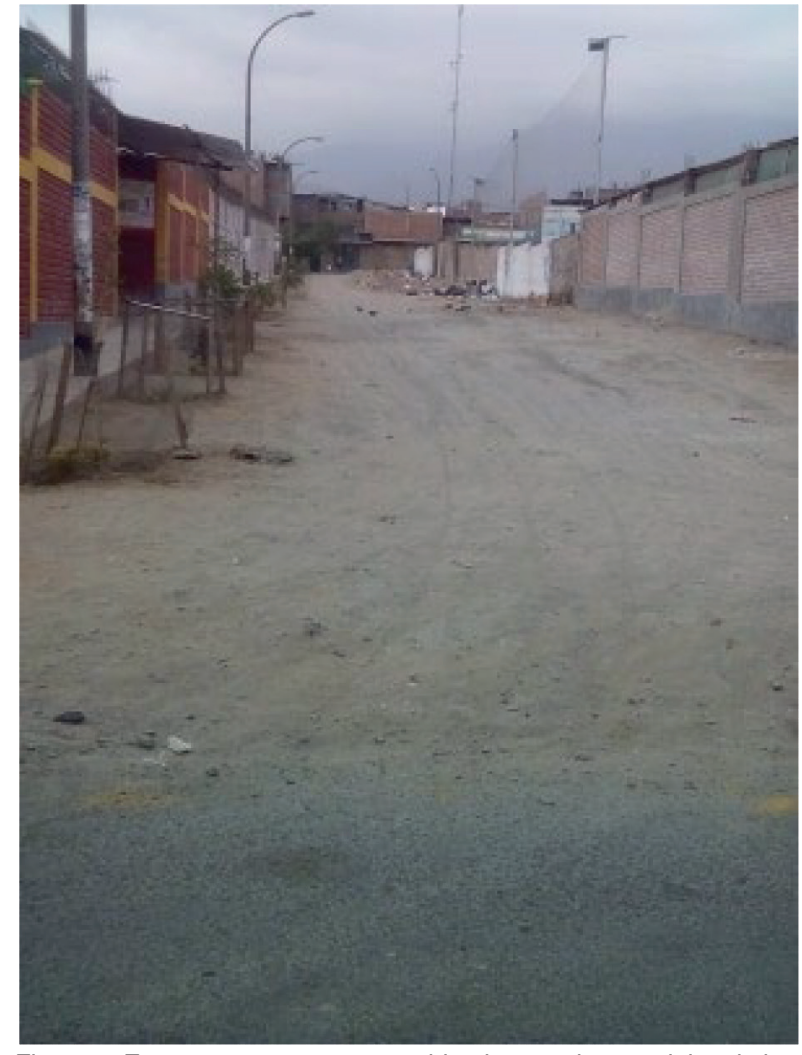

Figura 2. Este sector se encuentra ubicado entre las paralelas de los grupos habitacionales $\mathrm{N} .{ }^{\circ} 5$ y N.${ }^{\circ} 1$.

en la percepción de los sectores populares, que desnaturaliza la integración social y política. Además, se han privilegiado a ciertos grupos poderosos, de forma ilegal, en el manejo de obras públicas, lo que creó lazos clientelares. La ilegalidad institucional ha hecho emerger formas burocráticas para privilegiar obras locales y ello ha separado a los sujetos del municipio.

Dentro de este marco, el municipio no representa la parte fundamental de la descentralización, sino que repercute negativamente en la descentralización que se quiere construir. Si se deja todo tipo de argolla política, se podría señalar que es un espacio mercantil en donde se tejen confabulaciones administrativas que complementan la existencia del mal gobierno. Por consiguiente, la construcción de pistas y veredas, en Cruz de Motupe, se refleja como una política a medias. En cambio, se debe trabajar en el propósito de construir una forma de vida mediante la diversidad para rescatar los saberes populares y una efectiva participación popular con capacidad de incidir en la política pública.

\section{1. ¿Cómo nace el proyecto vial?}

Los moradores del pueblo joven Cruz de Motupe habían manifestado, de modo colectivo, su inconformidad con el descuido en el asfaltado de sus calles, debido a que diariamente los estudiantes y las amas de casa tenían que realizar largas caminatas por vías polvorientas, para llegar a sus centros de estudio y laborales que afectaban la población colindante al generar acumulación de desechos. Dichos espacios eran aprovechados por personas de mal vivir, entre ellos los drogadictos, por lo que los vecinos y los transeúntes eran víctimas de constantes asaltos. La condición desfavorable de la calle ponía en riesgo la salud de las personas.

En este orden de ideas, la obra se orientó a mejorar la transitabilidad vehicular y peatonal, pero también a reducir las enfermedades infectorespiratorias producidas por el polvo. En tal sentido, los dirigentes sociales participaron en el Presupuesto Participativo realizado en el 2010, al solicitar la pavimentación de sus calles, debido a que era una necesidad insatisfecha y sentida por muchos años. En el 2011 se convocó a una asamblea extraordinaria en la comunidad, en donde, por mayoría, se acordó la ejecución e implementación de la obra, y con este avance, los representantes de la JDC, a cargo del señor Roberto Lloclla Quispe, fueron con los arquitectos Juana María del Pilar Delgado Agurto y Edison Fernando Bravo Silva, responsables de la Gerencia de Desarrollo Urbano, y con los ingenieros Gabriel Franco Flores y Richard Mamani Apaza, responsables de la obra; durante septiembre, octubre, noviembre y diciembre del 2011.

Por otra parte, la Oficina de Programación e Inversión (OPI) del municipio hizo viable el proyecto de pistas y veredas, a partir de la elaboración del expediente técnico. Así, la obra nació como resultado de una necesidad de la población representada por sus dirigentes, la cual contó con el apoyo del municipio. Sin embargo, la ejecución de la obra no llegó a concluirse en algunas calles y no ha generado satisfacción en los sectores populares, puesto que la burocracia estatal, por medio de su uso coercitivo, ha violado los criterios del expediente técnico. En última instancia, no se ha podido construir la alternativa de desarrollo local, pues en la práctica implica un discurso hegemónico eurocéntrico; su incidencia ha operado en el marco del poder moderno y ha quebrado la organi- 
Tabla 3. Junta Directiva Central (2011)

\begin{tabular}{|l|l|}
\hline Presidente & Roberto Lloclla \\
\hline Vicepresidente & Rolando Tovar Baca \\
\hline Secretario de Actas y Archivos & Mauro Ticllacuri Ramos \\
\hline Secretario de Organización & Luis Nazario Arenaza Taipe \\
\hline Secretaria de Economía & Luz Violeta Julca Flores \\
\hline $\begin{array}{l}\text { Secretario de Defensa y } \\
\text { Disciplina }\end{array}$ & Manuel Augusto Silva More \\
\hline $\begin{array}{l}\text { Secretario de Relaciones } \\
\text { Publicas y Difusión }\end{array}$ & Valerio Polanco Ccoyllo \\
\hline $\begin{array}{l}\text { Secretario de Cultura y } \\
\text { Recreación }\end{array}$ & Oscar Arnaldo Chiscul Gómez \\
\hline $\begin{array}{l}\text { Secretaria de Salud y } \\
\text { Asistencia Social }\end{array}$ & $\begin{array}{l}\text { Alejandrina Mila Aldave } \\
\text { Dolores }\end{array}$ \\
\hline Fiscal & Rafael Campodónico Contreras \\
\hline
\end{tabular}

Fuente: Elaboración propia

zación comunal para el control y la fiscalización de obras públicas.

\subsection{Aliados estratégicos}

El primer aliado estratégico fue el PIMBP del MVCS. Su responsabilidad principal consistió en brindar asistencia técnica y apoyo en el proceso de implementación del proyecto, así como el cofinanciamiento para la ejecución de las obras. La etapa de inversión estuvo a cargo del MVCS, quien llevó a cabo las obras a través del Programa La Calle de Mi Barrio, el cual contaba con una unidad ejecutora organizada y dedicada a dicha función social. Estuvo representado por su director ejecutivo, el señor Moisés Orlando López Castro, con facultades otorgadas a través de las resoluciones ministeriales N. ${ }^{\circ} 001$ y 004-2011-Vivienda.

De este modo, su incidencia en el grupo habitacional N. ${ }^{o} 5$ del pueblo joven Cruz de Motupe fue determinante en la construcción de pistas y veredas para contribuir a mejorar, de manera íntegra, la calidad de vida de la población con déficits de infraestructura de servicios básicos y equipamiento, al promover el fortalecimiento institucional de los gobiernos locales y su coparticipación, así como la de la comunidad.

El segundo aliado estratégico fue la Municipalidad Distrital de San Juan de Lurigancho, que estuvo representada por el alcalde Carlos José Burgos Horna (2007-2014). Su responsabilidad era brindar una infraestructura adecuada, conforme con el Plan de
Desarrollo Concertado para la declaración de viabilidad del perfil técnico, según los criterios del Sistema Nacional de Inversión Pública y el trámite ante el MVCS para su inclusión en el Programa La Calle de Mi Barrio, y en segundo lugar, asumir el compromiso y la responsabilidad de la identificación de los sectores en donde se ejecutaría el proyecto social, mientras precisaba el nombre de las calles y el número a intervenir. Además, contaba con una Unidad Formuladora, que ha elaborado estudios de preinversión de diversos tipos de proyectos de inversión pública desde el 2004, y con una OPI, la cual tenía disponibilidad de financiamiento, operación y mantenimiento a lo largo del ciclo del proyecto, de acuerdo con las competencias y las facultades municipales.

La sostenibilidad institucional se desenvolvió en una lógica de compromiso con la Gerencia de Desarrollo Urbano y Subgerencia de Inversión Pública, para la construcción de la infraestructura vial de las principales calles de los grupos habitacionales N.o 1, 2, 3, 4, 5, 6 y 7 del pueblo joven Cruz de Motupe. Para ello se contaba con recursos profesionales y técnicos, y con la participación de manera activa de los vecinos beneficiarios, quienes eran motivados a cuidar la infraestructura urbana disponible (Fuente: Subgerencia de Inversión Pública de la Municipalidad de San Juan de Lurigancho, 2011).

El tercer aliado estratégico fue la población barrial que participó en el cofinanciamiento del proyecto, al aportar la mano de obra para el resane y la pintura de la fachada correspondiente a su inmueble, así como el mantenimiento del área verde frente a su propiedad, y la operación y el mantenimiento de los servicios y equipamiento (Fuente, Convenio de Financiamiento N.o 278-2022-MVCS). Es decir, la comunidad se comprometió con participar directamente en la gestión de la obra y en el proceso de fiscalización de esta. Sin embargo, es importante señalar que la JDC, los comités de calles, el Comité de Seguridad Ciudadana, el Comité de Parque y la Junta Directiva de los Grupos Habitacionales no tuvieron participación activa en la dirección del proyecto, sino que fue manejado y orientado por el Comité de Obra, pues en la realidad fueron una suerte de brókeres responsables de los costos de la acción colectiva para establecer vínculos con el municipio (Tanaka, 2001:18); además, su papel de interlocutor se enfrascó en la sustentabilidad de la obra. 


\subsection{Los costos del proyecto}

Según la Unidad Ejecutora de la Subgerencia de Inversiones Públicas de la Municipalidad de San Juan de Lurigancho, se establecieron los costos sociales y la población beneficiaria de la siguiente manera:

- La construcción de infraestructura vial y peatonal alrededor de las Mz. A hasta I, en las calles 1 al 6, 8, 9 y la Av. Cruz de Motupe Tramo CA. 3 a CA. 6 benefició a 1270 habitantes. El monto viable aprobado fue de 2032205 nuevos soles.

- La construcción de infraestructura vial, peatonal, áreas verdes y equipamiento urbano de la calle 62 Tramo CA. 29 - Av. Próceres de la Independencia, y la calle y Tramo CA. 48-CA. 29, pueblo joven Cruz de Motupe, estuvo a cargo de la Unidad Formuladora de la Municipalidad de San Juan de Lurigancho. Se beneficiaron 2952 personas. El monto viable aprobado fue de 808606.31 nuevos soles.

- La construcción de pistas y veredas en las calles internas del grupo habitacional N. 3 estuvo a cargo de la Unidad Formuladora el municipio de San Juan de Lurigancho. Se beneficiaron 2874 personas y el monto viable aprobado fue 2592052 nuevos soles.

- La construcción de infraestructura vial, peatonal y de seguridad en las calles del grupo habitacional N. ${ }^{\circ} 4$ estuvo a cargo de la Gerencia de Desarrollo Urbano de la Municipalidad de San Juan de Lurigancho. Se beneficiaron 1745 personas. El monto viable aprobado fue 2043855 nuevos soles.

- La construcción de pistas y veredas del grupo habitacional N. 5 estuvo a cargo de la Unidad Ejecutora de la Municipalidad de San Juan de Lurigancho, con participación directa del PIMBP. Las calles intervenidas fueron: 6, 34, 35, 36, 37, 38, 39, 40 y 41. Otras calles: 32, 31, 24, 26, 27, 29, 30, 33, 28 y Av. Cruz de Motupe, con un área de $16123 \mathrm{~m}^{2}$ de pavimento flexible, $10172.88 \mathrm{~m}^{2}$ de veredas, $5640.71 \mathrm{ml}$ de sardineles sumergidos, $824.44 \mathrm{~m}^{2}$ de señalización, $278.20 \mathrm{~m}^{2}$ de rampas, $6758.57 \mathrm{~m}^{2}$ de áreas verdes y $43 \mathrm{ml}$ del muro de mampostería de piedra.
En este caso, el MVCS transfirió recursos a favor de la municipalidad para el financiamiento de la ejecución del proyecto, por el monto total de 2775147.04 nuevos soles, incluidos los impuestos de ley, que comprenden 2707460.53 nuevos soles por concepto de obra y 67686.51 nuevos soles por concepto de supervisión, es decir, el $16 \%$.

De igual manera, la municipalidad aportó la suma de 555029.40 nuevos soles, equivalente al $16 \%$. Mientras que la población beneficiaria aportó la suma de 138757.35 nuevos soles, equivalente al $4 \%$. Por último, la población beneficiaria directa del proyecto fue de 198237 habitantes, cifra proyectada en función de los 49559 lotes de vivienda en las calles intervenidas.

- La construcción de pistas y veredas en las calles 44, 45, 46, 47, 49, 50, 51, 52, 53, 54, $67,68,69,70,71$ y Z del grupo habitacional No 6, tuvo como fuente de financiamiento los recursos ordinarios de la Gerencia de Desarrollo Urbano del Gobierno Local. Se beneficiaron 1898 personas y el monto del estudio definitivo o expediente técnico fue de 2313880 nuevos soles.

- En el grupo habitacional N. ${ }^{\circ} 7$ la ejecución del proyecto de pistas y veredas benefició directamente a 2262 habitantes, cifra proyectada de acuerdo con los 377 lotes de vivienda que se ubican cerca de las calles por ser intervenidas. Las vías que comprendieron las metas del proyecto fueron: $54,55,56,57,58$, 62, 63, 64, 51, 59, 53, 52, 60, 72, 71, 68, 69, 70, Pasaje San Juan y Av. Corazón de Jesús. El costo del proyecto fue de 3425212.55 nuevos soles y comprendió las vías con asfalto, construcción de veredas de losa de concreto, sardineles y señalización. El proyecto se hizo viable por la OPI de la Municipalidad de San Juan de Lurigancho.

Finalmente, la selección del proyecto y los costos sociales estuvieron dirigidos por funcionarios y autoridades locales que no representaron un instrumento decisivo en el involucramiento de la población para la fiscalización de la obra. Este hecho hace suponer que los actores externos aplicaron la maniobra de mani- 
pular información relevante, por lo que este proceso se denominaría la intervención de la dependencia con circuitos clandestinos.

\subsection{Niveles organizativos de la población en el ciclo del proyecto}

La población estuvo representada por el Comité de Obra, como una élite popular de interlocutores que se involucraron en relaciones de dominación y dependencia con los actores externos. Los criterios de la política de obra pública habían sido impuestos para mantener la pasividad de esa organización, pero al final se inmiscuyeron en redes clientelares por las autoridades locales. Esta situación alimentó las desigualdades y la exclusión popular en el proyecto de infraestructura social de corte asistencialista. A su vez, el Comité de Obra ha guiado el proyecto de pistas y veredas, pero estableció distanciamiento de coordinación con las demás organizaciones, como la JDC, la Junta Directiva de Grupos Habitacionales, el Comité de Parque, el Consejo de Fiscalización y el Comité de Seguridad Ciudadana.

La incidencia de actores externos, el débil rol del Comité de Obra y la apatía del nuevo sector popular ha permitido la constitución de grupos de intereses. Al mismo tiempo, la distorsión organizativa ha hecho posible que los actores políticos manipulen las obras públicas en un desborde sin resultados. Estas no deben partir de la visión tecnocrática, sino de las necesidades de los sujetos sociales. Sin duda, la participación de la población se realizó en tres ciclos:

\section{Conclusión}

En Cruz de Motupe, la participación popular se desenvuelve como un sistema de negociación en un proceso dependiente, y su fragilidad obliga a optar por acciones individualistas. En este sentido, se ha perdido la proyección política como resultado de la crisis del Estado y de las instituciones. Sin duda, una participación popular transcultural, entendida como una alternativa histórica en la diversidad, libertad e igualdad para elevar la capacidad de acción de la sociedad sobre sí misma y sobre su entorno, debe ser el camino emancipatorio para la transformación social, a partir de una lógica de cooperación y solidaridad, con el fin de dar el primer paso al sentido de la ciudadanía y cimentar la base de un régimen representativo popular.

Dentro de este marco, la nueva propuesta participativa apunta a la intervención de distintos actores en la definición de las metas de acción de una colectividad y de los medios para alcanzarlas en todos los espacios donde se despliegan las relaciones humanas, y cuya incidencia es mayor o menor según los intereses, valoraciones y las percepciones de los involucrados en la interacción, para asegurar y fiscalizar que las obras ejecutadas en la comunidad tengan conservación y mantenimiento. Sin embargo, se hace necesario enfatizar en que la participación popular en zonas consolidadas se ha encontrado atomizada por las estructuras dominantes, y urge una profunda descolonización del patrón de poder para afirmar una participación, en creciente integración, como una cultura civilizatoria de vida. De eso se desprende que no se ha consolidado una participación integrada y pluralista desde los actores.

TABla 4. Fases de participación

\begin{tabular}{|c|c|c|}
\hline Pre inversión & Inversión & Posinversión \\
\hline $\begin{array}{l}\text { La población identificó las necesidades } \\
\text { para seleccionar el proyecto. Se eligió } \\
\text { al Comité de Obra, integrado por el } \\
\text { presidente, el secretario, la tesorera y el } \\
\text { fiscal, para representar los intereses de la } \\
\text { comunidad y la viabilidad de la obra. Su } \\
\text { desenvolvimiento en la esfera pública fue } \\
\text { agonizante y dependiente. }\end{array}$ & $\begin{array}{l}\text { Se puso en conocimiento de la población } \\
\text { los gastos, el manejo de dinero, el avance } \\
\text { físico de la obra y el proceso de rendición } \\
\text { de cuentas. Este ejercicio tuvo muchos } \\
\text { vacíos. El } 70 \% \text { de las personas abonaron } \\
\text { un monto entre } 200 \text { a } 500 \text { soles por } \\
\text { casa y el } 30 \% \text { se negaron a pagar. La } \\
\text { inexistencia de rendición de cuentas } \\
\text { generó divisionismos y desconfianza con } \\
\text { las autoridades locales. }\end{array}$ & $\begin{array}{l}\text { Se inició el proceso de mantenimiento } \\
\text { y conservación de la obra, con base en } \\
\text { un plan de trabajo y la vigilancia de } \\
\text { su cumplimiento. Es menester señalar } \\
\text { que los pobladores no administraron el } \\
\text { dinero, sino el municipio, para la compra } \\
\text { de materiales y el pago a los profesionales } \\
\text { y obreros. }\end{array}$ \\
\hline
\end{tabular}

Fuente: elaboración propia 
De este modo, la participación transcultural se orienta a un proceso de cambio social y se constituye en la democratización del espacio público para fortalecer las capacidades autorganizativas de la sociedad. Esto obedece a la existencia de un proyecto con voluntad política real que universalice a los sectores populares. En este sentido, se abre la posibilidad de un cambio en las relaciones de poder entre los ciudadanos y las instituciones públicas, a fin de que sean tomados en cuenta para la implementación de programas y proyectos en beneficio de la calidad de vida. Aunque la demanda de la participación se ha convertido en un móvil de la acción pública que se inscribe en un contexto de creciente conflictividad social, cada vez surgen más grupos constituidos sobre una base territorial, y sus representantes se mantienen deliberadamente fuera de las instancias de mediación y representación tradicional. Para tal efecto, la participación como forma política y social no puede estar separada de los sujetos sociales, por lo que su expresión involucra una postura cultural civilizatoria para negar toda dominación instrumentalista o fundamentalista. Es por esta razón que la participación transcultural afirma una cultura y política de vida, en todas sus formas, al servicio de los humanos, concebida como un todo para plantear y resolver los problemas globales y específicos. Así, su carácter conduce a una voluntad política abierta a la diversidad de los pueblos, contra el poder imperial hegemónico.

\section{Bibliografía}

Alza Barco, Carlos (2008). Conflictividad en el Perú: una lectura desde las políticas públicas. En El papel de las politicas públicas. Lima: Fondo Editorial PUCP.

BÉJAR, Héctor (2007). Política social, justicia social. Lima: Editorial CEDEP.
Estrella Niño, Elías (2008). Toma de tierra y fundación del asentamiento humano Cruz de Motupe. Lima: Impresión Juan Gutenberg Editores.

Lynch, Nicolás (2017). Populismo ¿Dictadura o democracia? Lima: Fondo Editorial de la Facultad de Ciencias Sociales UNMSM.

Mejía Navarrete, Julio (1990). Estado y municipio en el Perú. Lima: Consejo Nacional de Ciencia y Tecnología.

Mejía Navarrete, Julio (2014). Sociedad, consumo y ética. El Perú en tiempos de la globalización. Fondo editorial, Facultad de Ciencias Sociales de la UNMSM.

Panfichi, Aldo y Coronel, Omar (2014). La relación entre sociedad y política en Perú y Bolivia. En Conflicto social en los Andes. Lima: Fondo editorial PUCP.

Municipalidad de San Juan de Lurigancho (2011). Proyecto de inversión pública a nivel de perfil. Construcción de pistas y veredas del pueblo joven Cruz de Motupe. Lima: Gerencia de Desarrollo Urbano.

Ríos Burga, Jaime (2011). El quehacer sociológico en América Latina. Un diálogo teórico con sus actores. Lima: Universidad Nacional Mayor de San Marcos.

Quijano, Aníbal (2014). Cuestiones y horizontes: de la dependencia histórico-estructural a la colonialidad/descolonialidad del poder (antologías). Selección a cargo de Danilo Assis Clímaco. Ciudad Autónoma de Buenos Aires: CLACSO.

Quijano, Aníbal (2009). Descolonialidad del poder. El horizonte alternativo. En Plurinacionalidad. Democracia en la diversidad. Quito: Impreso en Quito-Ecuador.

Quiroz, W. Alfonso (2013). Historia de la corrupción en el Perú. Lima: IEP.

Tanaka, Martín (2001). Participación popular en políticas sociales: cuando puede ser democrática y eficiente, y cuando todo lo contrario. Lima: Consorcio de Investigación Económica y Social / IEP. 